\title{
Existence and Stability of Antiperiodic Solution for a Class of Generalized Neural Networks with Impulses and Arbitrary Delays on Time Scales
}

\author{
Yongkun Li, Erliang Xu, and Tianwei Zhang \\ Department of Mathematics, Yunnan University, Kunming, Yunnan 650091, China \\ Correspondence should be addressed to Tianwei Zhang, 1200801347@stu.ynu.edu.cn \\ Received 14 June 2010; Accepted 16 August 2010 \\ Academic Editor: Kok Lay Teo
}

Copyright $@ 2010$ Yongkun Li et al. This is an open access article distributed under the Creative Commons Attribution License, which permits unrestricted use, distribution, and reproduction in any medium, provided the original work is properly cited.

By using coincidence degree theory and Lyapunov functions, we study the existence and global exponential stability of antiperiodic solutions for a class of generalized neural networks with impulses and arbitrary delays on time scales. Some completely new sufficient conditions are established. Finally, an example is given to illustrate our results. These results are of great significance in designs and applications of globally stable anti-periodic Cohen-Grossberg neural networks with delays and impulses .

\section{Introduction}

In this paper, we consider the following generalized neural networks with impulses and arbitrary delays on time scales:

$$
\begin{aligned}
x^{\Delta}(t) & =A(t, x(t))\left[B(t, x(t))+F\left(t, x_{t}\right)\right], \quad t \in \mathbb{T}, t \neq t_{k}, \\
\Delta x\left(t_{k}\right) & =x\left(t_{k}^{+}\right)-x\left(t_{k}^{-}\right)=I_{k}\left(x\left(t_{k}\right)\right), \quad t=t_{k}, k \in \mathbb{N},
\end{aligned}
$$

where $\mathbb{T}$ is an $\omega / 2$-periodic time scale and if $t \in \mathbb{T}, \theta \in E$, then $t+\theta \in \mathbb{T}, E$ is a subset of $R^{-}=(-\infty, 0], A(t, x(t))=\operatorname{diag}\left(a_{1}\left(t, x_{1}(t)\right), a_{2}\left(t, x_{2}(t)\right), \ldots, a_{n}\left(t, x_{n}(t)\right)\right), B(t, x(t))=$ $\left(b_{1}\left(t, x_{1}(t)\right), b_{2}\left(t, x_{2}(t)\right), \ldots, b_{n}\left(t, x_{n}(t)\right)\right)^{T}, F\left(t, x_{t}\right)=\left(f_{1}\left(t, x_{t}\right), \ldots, f_{n}\left(t, x_{t}\right)\right)^{T}, f_{i}\left(t, x_{t}\right)=$ $f_{i}\left(t, x_{1 t}, x_{2 t}, \ldots, x_{n t}\right), x_{i t}(\theta)=x_{i}(t+\theta), t \in \mathbb{T}, \theta \in E, i=1,2, \ldots, n$, and $x\left(t_{k}^{+}\right), x\left(t_{k}^{-}\right)$ represent the right and left limits of $x\left(t_{k}\right)$ in the sense of time scales, $\left\{t_{l}\right\}$ is a sequence of real numbers such that $0<t_{1}<t_{2}<\cdots<t_{n} \rightarrow \infty$ as $l \rightarrow \infty$. There exists a positive integer $q$ such that $t_{l+q}=t_{l}+\omega / 2, I_{k+q}(u)=-I_{k}(-u), \quad l \in \mathbb{Z}, u \in \mathbb{R}$. Without loss of generality, we also 
assume that $[0, \omega / 2)_{\mathbb{T}} \cap\left\{t_{l}: l \in \mathbb{N}\right\}=\left\{t_{1}, t_{2}, \ldots, t_{q}\right\}$. For each interval $I$ of $\mathbb{R}$, we denote that $I_{\mathbb{T}}=I \cap \mathbb{T}$, especially, we denote that $\mathbb{T}^{+}=\mathbb{T} \cap[0,+\infty)$.

System (1.1) includes many neural continuous and discrete time networks [1-9]. For examples, the high-order Hopfield neural networks with impulses and delays (see [8]):

$$
\begin{aligned}
x_{i}^{\prime}(t)=-a_{i}\left(x_{i}(t)\right)[ & b_{i}\left(x_{i}(t)\right)-\sum_{j=1}^{n} a_{i j}(t) g_{j}\left(x_{j}(t)\right)-\sum_{j=1}^{n} b_{i j}(t) g_{j}\left(x_{j}\left(t-\tau_{j}(t)\right)\right) \\
& \left.-\sum_{j=1}^{n} \sum_{l=1}^{n} b_{i j l}(t) g_{j}\left(x_{j}\left(t-\tau_{j}(t)\right)\right) g_{l}\left(x_{l}\left(t-\tau_{l}(t)\right)\right)+I_{i}(t)\right], \quad t \neq t_{k}, \\
\Delta x_{i}\left(t_{k}\right)= & x_{i}\left(t_{k}^{+}\right)-x_{i}\left(t_{k}^{-}\right)=e_{i k}\left(x_{i}\left(t_{k}\right)\right), \quad i=1,2, \ldots, n, k=1,2, \ldots
\end{aligned}
$$

the Cohen-Grossberg neural networks with bounded and unbounded delays (see [9]):

$$
\begin{array}{r}
x_{i}^{\prime}(t)=-a_{i}\left(x_{i}(t)\right)\left[b_{i}\left(x_{i}(t)\right)-\sum_{j=1}^{\mathrm{n}} c_{i j}(t) f_{j}\left(x_{j}(t)\right)-\sum_{j=1}^{n} c_{i j}(t) g_{j}\left(x_{j}\left(t-\tau_{i j}(t)\right)\right)\right. \\
\left.\quad-\sum_{j=1}^{n} d_{i j}(t) h_{j}\left(\int_{0}^{\infty} K_{i j}(u) x_{j}(t-u) \mathrm{d} u\right)+I_{i}(t)\right], \quad t \neq t_{k}, \\
\Delta x_{i}\left(t_{k}\right)=x_{i}\left(t_{k}^{+}\right)-x_{i}\left(t_{k}^{-}\right)=l_{i k}\left(x_{i}\left(t_{k}\right)\right), \quad i=1,2, \ldots, n, \quad k=1,2, \ldots,
\end{array}
$$

and so on.

Arising from problems in applied sciences, it is well known that anti-periodic problems of nonlinear differential equations have been extensively studied by many authors during the past twenty years; see [10-21] and references cited therein. For example, antiperiodic trigonometric polynomials are important in the study of interpolation problems $[22,23]$, and anti-periodic wavelets are discussed in [24].

Recently, several authors [25-30] have investigated the anti-periodic problems of neural networks without impulse by similar analytic skills. However, to the best of our knowledge, there are few papers published on the existence of anti-periodic solutions to neural networks with impulse.

The main purpose of this paper is to study the existence and global exponential stability of anti-periodic solutions of system (1.1) by using the method of coincidence degree theory and Lyapunov functions.

The initial conditions associated with system (1.1) are of the form

$$
x_{0}=\phi, \quad \text { that is, } x_{i}(\theta)=\phi_{i}(\theta), \quad \theta \in E, \quad i=1,2, \ldots, n \text {. }
$$

Throughout this paper, we assume that

$\left(H_{1}\right) a_{i}(t, u) \in C\left(\mathbb{T} \times \mathbb{R}, \mathbb{R}^{+}\right), a_{i}(t+\omega / 2,-u)=a_{i}(t, u)$, and there exist positive constants $a_{i}^{m}, a_{i}^{M}$ such that $0<a_{i}^{m}<a_{i}(t, u)<a_{i}^{M}$ for all $t \in \mathbb{T}, u \in \mathbb{R}, i=1,2, \ldots, n$; 
$\left(H_{2}\right) b_{i}(t, u) \in C(\mathbb{T} \times \mathbb{R}, \mathbb{R}), b_{i}(t+\omega / 2,-u)=-b_{i}(t, u)$. There exist positive constants $\mu_{i}$ and $L_{i}^{b}$ such that

$$
\frac{\partial b_{i}(t, u)}{\partial u} \geq \mu_{i}, \quad\left|b_{i}(t, u)-b_{i}(t, v)\right| \leq L_{i}^{b}|u-v|, \quad b_{i}(t, 0)=0,
$$

for all $t \in \mathbb{T}, u, v \in \mathbb{R}, i=1,2, \ldots, n$;

$\left(H_{3}\right) f_{i} \in C\left(\mathbb{T} \times \mathbb{R}^{n}, \mathbb{R}\right), f_{i}(t+\omega / 2,-u)=-f_{i}(t, u)$, for $i=1,2, \ldots, n$. There exist positive constants $c_{i}$ such that

$$
\left|f_{i}\left(t, x_{1 t}, \ldots, x_{n t}\right)-f_{i}\left(t, y_{1 t}, \ldots, y_{n t}\right)\right| \leq c_{i} \sum_{j=1}^{n}\left|x_{j t}-y_{j t}\right|
$$

for all $\left(t, x_{1 t}, \ldots, x_{n t}\right),\left(t, y_{1 t}, \ldots, y_{n t}\right) \in \mathbb{T} \times \mathbb{R}^{n}$ and $f_{i}(t, 0, \ldots, 0)=0, \quad i=1,2, \ldots, n$;

$\left(H_{4}\right) I_{i k} \in C(\mathbb{R}, \mathbb{R})$ and there exist positive constants $L_{i k}^{I}$ such that

$$
\left|I_{i k}(u)-I_{i k}(v)\right| \leq L_{i k}^{I}|u-v|,
$$

for all $u, v \in \mathbb{R}, k \in \mathbb{N}, i=1,2, \ldots, n$.

For convenience, we introduce the following notation:

$$
h^{M}=\max _{t \in[0, \omega]_{\mathbb{T}}}|h(t)|, \quad h^{m}=\min _{t \in[0, \omega]_{\mathbb{T}}}|h(t)|, \quad\|h\|_{2}=\left(\int_{0}^{\omega}|h(t)|^{2} \Delta t\right)^{1 / 2},
$$

where $h$ is an $\omega$-periodic function.

The organization of this paper is as follows. In Section 2, we introduce some definitions and lemmas. In Section 3, by using the method of coincidence degree theory, we obtain the existence of the anti-periodic solutions of system (1.1). In Section 4, we give the criteria of global exponential stability of the anti-periodic solutions of system (1.1). In Section 5, an example is also provided to illustrate the effectiveness of the main results in Sections 3 and 4. The conclusions are drawn in Section 6.

\section{Preliminaries}

In this section, we will first recall some basic definitions and lemmas which can be found in books [31, 32].

Definition 2.1 (see [31]). A time scale $\mathbb{T}$ is an arbitrary nonempty closed subset of real numbers $\mathbb{R}$. The forward and backward jump operators $\sigma, \rho: \mathbb{T} \rightarrow \mathbb{T}$ and the graininess $\mu: \mathbb{T} \rightarrow \mathbb{R}^{+}$ are defined, respectively, by

$$
\sigma(t):=\inf \{s \in \mathbb{T}: s>t\}, \quad \rho(t):=\sup \{s \in \mathbb{T}: s<t\}, \quad \mu(t)=\sigma(t)-t .
$$


Definition 2.2 (see [31]). A function $f: \mathbb{T} \rightarrow \mathbb{R}$ is called right-dense continuous provided it is continuous at right-dense point of $\mathbb{T}$ and left-side limit exists (finite) at left-dense point of $\mathbb{T}$. The set of all right-dense continuous functions on $\mathbb{T}$ will be denoted by $C_{\mathrm{rd}}=C_{\mathrm{rd}}(\mathbb{T})=$ $C_{\mathrm{rd}}(\mathbb{T}, \mathbb{R})$. If $f$ is continuous at each right-dense and left-dense point, then $f$ is said to be a continuous function on $\mathbb{T}$, the set of continuous function will be denoted by $C(\mathbb{T})$.

Definition 2.3 (see [31]). For $x: \mathbb{T} \rightarrow \mathbb{R}$, one defines the delta derivative of $x(t), x^{\Delta}(t)$ to be the number (if it exists) with the property that for a given $\varepsilon>0$, there exists a neighborhood $U$ of $t$ such that

$$
\left|[x(\sigma(t))-x(t)]-x^{\Delta}(t)[\sigma(t)-s]\right| \leq \varepsilon|\sigma(t)-s|,
$$

for all $s \in U$.

Definition 2.4 (see [31]). If $F^{\Delta}(t)=f(t)$, then one defines the delta integral by

$$
\int_{a}^{t} f(s) \Delta s=F(t)-F(a)
$$

Definition 2.5 (see [33]). For each $t \in \mathbb{T}$, let $N$ be a neighborhood of $t$. Then, one defines the generalized derivative (or dini derivative), $D^{+} u^{\Delta}(t)$ to mean that, given $\varepsilon>0$, there exists a right neighborhood $N(\varepsilon) \subset N$ of $t$ such that

$$
\frac{u(\sigma(t))-u(s)}{\mu(t, s)}<D^{+} u^{\Delta}(t)+\varepsilon,
$$

for each $s \in N(\varepsilon), s>t$, where $\mu(t, s)=\sigma(t)-s$.

In case $t$ is right-scattered and $u(t)$ is continuous at $t$, this reduces to

$$
D^{+} u^{\Delta}(t)=\frac{u(\sigma(t))-u(t)}{\sigma(t)-t}
$$
following.

Similar to [34], we will give the definition of anti-periodic function on a time scale as

Definition 2.6. Let $\mathbb{T} \neq \mathbb{R}$ be a periodic time scale with period $p$. One says that the function $f: \mathbb{T} \rightarrow \mathbb{R}$ is $\omega / 2$-anti-periodic if there exists a natural number $n$ such that $\omega / 2=n p$, $f(t+\omega / 2)=-f(t)$ for all $t \in \mathbb{T}$ and $\omega$ is the smallest number such that $f(t+\omega / 2)=-f(t)$.

If $\mathbb{T}=\mathbb{R}$, one says that $f$ is $\omega / 2$-anti-periodic if $\omega / 2$ is the smallest positive number such that $f(t+\omega / 2)=-f(t)$ for all $t \in \mathbb{T}$.

Definition 2.7 (see [31]). A function $p: \mathbb{T} \rightarrow \mathbb{R}$ is called regressive if $1+\mu(t) p(t) \neq 0$ for all $t \in \mathbb{T}^{k}$, where $\mu(t)=\sigma(t)-t$ is the graininess function. If $p$ is regressive and right-dense continuous function, then the generalized exponential function $e_{p}$ is defined by

$$
e_{p}(t, s)=\exp \left\{\int_{s}^{t} \xi_{\mu(\tau)}(p(\tau)) \Delta \tau\right\}
$$


for $s, t \in \mathbb{T}$, with the cylinder transformation

$$
\xi_{h}(z)= \begin{cases}\frac{\log (1+h z)}{h}, & \text { if } h \neq 0, \\ z, & \text { if } h=0 .\end{cases}
$$

Let $p, q: \mathbb{T} \rightarrow \mathbb{R}$ be two regressive functions, we define

$$
p \oplus q:=p+q+\mu p q, \quad \ominus p:=-\frac{p}{1+\mu p}, \quad p \ominus q=p \oplus(\ominus q)
$$

Then the generalized exponential function has the following properties.

Lemma 2.8 (see $[31,32]$ ). Assume that $p, q: \mathbb{T} \rightarrow \mathbb{R}$ are two regressive functions, then

(i) $e_{0}(t, s) \equiv 1$ and $e_{p}(t, t) \equiv 1$;

(ii) $e_{p}(\sigma(t), s)=(1+\mu(t) p(t)) e_{p}(t, s)$;

(iii) $e_{p}(t, \sigma(s))=e_{p}(t, s) /(1+\mu(s) p(s))$;

(iv) $1 / e_{p}(t, s)=e_{\ominus p}(t, s)$;

(v) $e_{p}(t, s)=1 / e_{p}(s, t)=e_{\ominus p}(s, t)$;

(vi) $e_{p}(t, s) e_{p}(s, r)=e_{p}(t, r)$;

(vii) $e_{p}^{\Delta}(\cdot, s)=p e_{p}(\cdot, s)$.

Lemma 2.9 (see [31]). Assume that $f, g: \mathbb{T} \rightarrow \mathbb{R}$ are delta differentiable at $t \in \mathbb{T}^{k}$. Then

$$
(f g)^{\Delta}(t)=f^{\Delta}(t) g(t)+f(\sigma(t)) g^{\Delta}(t)=f(t) g^{\Delta}(t)+f^{\Delta}(t) g(\sigma(t)) .
$$

The following lemmas can be found in $[35,36]$, respectively.

Lemma 2.10. Let $t_{1}, t_{2} \in[0, w]_{\mathbb{T}}$. If $x: \mathbb{T} \rightarrow \mathbb{R}$ is $\omega$-periodic, then

$$
x(t) \leq x\left(t_{1}\right)+\int_{0}^{\omega}\left|x^{\Delta}(s)\right| \Delta s, \quad x(t) \geq x\left(t_{2}\right)-\int_{0}^{\omega}\left|x^{\Delta}(s)\right| \Delta s .
$$

Lemma 2.11. Let $a, b \in \mathbb{T}$. For $r d$-continuous functions $f, g:[a, b] \rightarrow \mathbb{R}$, one has

$$
\int_{a}^{b}|f(t) g(t)| \Delta t \leq\left(\int_{a}^{b}|f(t)|^{2} \Delta t\right)^{1 / 2}\left(\int_{a}^{b}|g(t)|^{2} \Delta t\right)^{1 / 2}
$$

Definition 2.12. The anti-periodic solution $x^{*}(t)=\left(x_{1}^{*}(t), x_{2}^{*}(t), \ldots, x_{n}^{*}(t)\right)^{T}$ of system (1.1) is said to be globally exponentially stable if there exist positive constants $\epsilon$ and $M=M(\epsilon) \geq 1$, 
for any solution $x(t)=\left(x_{1}(t), x_{2}(t), \ldots, x_{n}(t)\right)^{T}$ of system (1.1) with the initial value $\phi(t)=$ $\left(\phi_{1}(t), \phi_{2}(t), \ldots, \phi_{n}(t)\right)^{T} \in C\left(E_{\mathbb{T}}, \mathbb{R}^{n}\right)$, such that

$$
\sum_{i=1}^{n}\left|x_{i}(t)-x_{i}^{*}(t)\right| \leq M(\epsilon) e_{\ominus \epsilon}(t, \alpha)\left\|\phi-x^{*}\right\|
$$

where

$$
\left\|\phi-x^{*}\right\|=\sum_{i=1}^{n} \sup _{s \in E_{\mathbb{T}}}\left|\phi_{i}(s)-x_{i}^{*}(s)\right|, \quad \alpha \in E_{\mathbb{T}} .
$$

The following continuation theorem of coincidence degree theory is crucial in the arguments of our main results.

Lemma 2.13 (see [37]). Let $\mathbb{X}, \mathbb{X}$ be two Banach spaces, $\Omega \subset \mathbb{X}$ be open bounded and symmetric with $0 \in \Omega$. Suppose that $L: D(L) \subset \mathbb{X} \rightarrow \mathbb{Y}$ is a linear Fredholm operator of index zero with $D(L) \cap \bar{\Omega} \neq \emptyset$ and $N: \bar{\Omega} \rightarrow \mathbb{Y}$ is L-compact. Further, one also assumes that

(H) $L x-N x \neq \lambda(-L x-N(-x))$ for all $x \in D(L) \cap \partial \Omega, \quad \lambda \in(0,1]$.

Then the equation $L x=N x$ has at least one solution on $D(L) \cap \bar{\Omega}$.

\section{Existence of Antiperiodic Solutions}

In this section, by using Lemma 2.13 , we will study the existence of at least one anti-periodic solution of (1.1).

Theorem 3.1. Assume that $\left(H_{1}\right)-\left(H_{4}\right)$ hold. Suppose further that

$\left(H_{5}\right) E=\left(e_{i j}\right)_{n \times n}$ is a nonsingular $M$ matrix, where, for $i, j=1,2, \ldots, n$,

$$
e_{i j}= \begin{cases}\omega a_{i}^{m}-\omega^{2} a_{i}^{m} a_{i}^{M} L_{i}^{b}-\omega a_{i}^{m} \sum_{k=1}^{2 q} L_{i k}^{I}-\frac{1}{\mu_{i}} \sum_{k=1}^{2 q} L_{i k}^{I}-\left(\frac{1}{\mu_{i}}+\omega a_{i}^{m}\right) a_{i}^{M} \omega c_{i}, & i=j, \\ -\left(\frac{1}{\mu_{i}}+\omega a_{i}^{m}\right) a_{i}^{M} \omega c_{i}, & i \neq j .\end{cases}
$$

Then system (1.1) has at least one $\omega / 2$-anti-periodic solution.

Proof. Let $C^{k}\left[0, \omega ; t_{1}, \ldots, t_{q}, t_{q+1}, \ldots, t_{2 q}\right]_{\mathbb{T}}=\left\{x:[0, \omega]_{\mathbb{T}} \rightarrow \mathbb{R}^{n+m} \mid x^{k}(t)\right.$ is a piecewise continuous map with first-class discontinuity points in $[0, \omega]_{\mathbb{T}} \cap\left\{t_{k}\right\}$, and at each discontinuity point it is continuous on the left $\}$. Take

$$
\begin{gathered}
\mathbb{X}=\left\{x \in C\left[0, \omega ; t_{1}, \ldots, t_{q}, t_{q+1}, \ldots, t_{2 q}\right]_{\mathbb{T}}: x\left(t+\frac{\omega}{2}\right)=-x(t), \forall t \in\left[0, \frac{\omega}{2}\right]_{\mathbb{T}}\right\}, \\
\mathbb{Y}=\mathbb{X} \times \mathbb{R}^{n \times q}
\end{gathered}
$$


Journal of Inequalities and Applications

are two Banach spaces with the norms

$$
\|x\| \mathbb{X}=\sum_{i=1}^{n}\left|x_{i}\right|_{0}, \quad\|z\| \mathbb{Y}=\|x\| \mathbb{X}+\|y\|
$$

respectively, where $\left|x_{i}\right|_{0}=\max _{t \in[0, \omega]_{\mathbb{T}}}\left|x_{i}(t)\right|, i=1, \ldots, n,\|\cdot\|$ is any norm of $\mathbb{R}^{n \times q}$.

Set

$$
L: \operatorname{Dom} \quad L \cap \mathbb{X} \longrightarrow \mathbb{Y}, \quad x \longrightarrow\left(x^{\Delta}, \Delta x\left(t_{1}\right), \ldots, \Delta x\left(t_{q}\right)\right),
$$

where

$$
\begin{gathered}
\operatorname{Dom} L=\left\{x \in C^{1}\left[0, \omega ; t_{1}, \ldots, t_{2 q}\right]_{\mathbb{T}}: x\left(t+\frac{\omega}{2}\right)=-x(t), \forall t \in\left[0, \frac{\omega}{2}\right]_{\mathbb{T}}\right\}, \\
N: \mathbb{X} \longrightarrow \mathbb{Y}, \\
N x=\left(\left(\begin{array}{c}
A_{1}(t) \\
\vdots \\
A_{n}(t)
\end{array}\right),\left(\begin{array}{c}
I_{11}\left(x_{1}\left(t_{1}\right)\right) \\
\vdots \\
I_{n 1}\left(x_{n}\left(t_{1}\right)\right)
\end{array}\right), \ldots,\left(\begin{array}{c}
I_{1 q}\left(x_{1}\left(t_{q}\right)\right) \\
\vdots \\
I_{n q}\left(x_{n}\left(t_{q}\right)\right)
\end{array}\right)\right),
\end{gathered}
$$

where

$$
A_{i}(t)=a_{i}\left(t, x_{i}(t)\right)\left[b_{i}\left(t, x_{i}(t)\right)+f_{i}\left(t, x_{t}\right)\right], \quad i=1,2, \ldots, n .
$$

It is easy to see that

$$
\operatorname{Ker} L=\{0\}, \quad \operatorname{Im} L=\left\{z=\left(f, C_{1}, \ldots, C_{q}\right) \in \mathbb{Y}: \int_{0}^{\omega} f(s) \Delta s=0\right\}=\mathbb{Y} .
$$

Thus, $\operatorname{dim} \operatorname{Ker} L=0=\operatorname{codim} \operatorname{Im} L$, and $L$ is a linear Fredholm mapping of index zero.

Define the projectors $P: \mathbb{X} \rightarrow \operatorname{Ker} L$ and $Q: \mathbb{Y} \rightarrow \mathbb{Y}$ by

$$
\begin{gathered}
P x=\int_{0}^{\omega} x(s) \Delta s=0, \\
Q z=Q\left(f, C_{1}, \ldots, C_{q}\right)=\left(\frac{1}{\omega} \int_{0}^{\omega} f(s) \Delta s, 0, \ldots, 0\right),
\end{gathered}
$$


respectively. It is not difficult to show that $P$ and $Q$ are continuous projectors such that

$$
\operatorname{Im} P=\operatorname{Ker} L, \quad \operatorname{Im} L=\operatorname{Ker} Q=\operatorname{Im}(I-Q) .
$$

Further, let $L_{P}^{-1}=L_{\mid \operatorname{Dom} L \cap K e r} P$ and the generalized inverse $K_{P}=L_{P}^{-1}$ is given by

$$
K_{P} z=\int_{0}^{t} f(s) \Delta s+\sum_{t>t_{k}} C_{k}-\frac{1}{2} \int_{0}^{\omega / 2} f(s) \Delta s-\frac{1}{2} \sum_{k=1}^{q} C_{k}
$$

in which $C_{q+i}=-C_{i}$ for all $1 \leq i \leq q$.

Similar to the proof of Theorem 3.1 in [38], it is not difficult to show that $Q N(\bar{\Omega})$, $K_{P}(I-Q) N(\bar{\Omega})$ are relatively compact for any open bounded set $\Omega \subset \mathbb{X}$. Therefore, $N$ is $L$-compact on $\bar{\Omega}$ for any open bounded set $\Omega \subset \mathbb{X}$.

Corresponding to the operator equation $L x-N x=\lambda(-L x-N(-x)), \lambda \in(0,1]$, we have

$$
\begin{aligned}
x^{\Delta}(t) & =\frac{1}{1+\lambda} G(t, x)-\frac{\lambda}{1+\lambda} G(t,-x), \quad t \in \mathbb{T}^{+}, t \neq t_{k}, \\
\Delta x\left(t_{k}\right) & =\frac{1}{1+\lambda} I_{k}\left(x\left(t_{k}\right)\right)-\frac{\lambda}{1+\lambda} I_{k}\left(-x\left(t_{k}\right)\right), \quad t=t_{k}, \quad k \in \mathbb{N},
\end{aligned}
$$

or

$$
\begin{gathered}
x_{i}^{\Delta}(t)=\frac{1}{1+\lambda} G_{i}(t, x)-\frac{\lambda}{1+\lambda} G_{i}(t,-x), \quad t \in \mathbb{T}^{+}, \quad t \neq t_{k} \\
\Delta x_{i}\left(t_{k}\right)=\frac{1}{1+\lambda} I_{i k}\left(x_{i}\left(t_{k}\right)\right)-\frac{\lambda}{1+\lambda} I_{i k}\left(-x_{i}\left(t_{k}\right)\right), \quad t=t_{k}, \quad i=1,2, \ldots, n, \quad k \in \mathbb{N},
\end{gathered}
$$

where

$$
\begin{gathered}
G_{i}(t, x)=a_{i}\left(t, x_{i}(t)\right)\left[b_{i}\left(t, x_{i}(t)\right)+f_{i}\left(t, x_{t}\right)\right], \\
G_{i}(t,-x)=a_{i}\left(t,-x_{i}(t)\right)\left[b_{i}\left(t,-x_{i}(t)\right)+f_{i}\left(t,-x_{t}\right)\right], \quad i=1,2, \ldots, n .
\end{gathered}
$$


Set $t_{0}=t_{0}^{+}=0, \quad t_{2 q+1}=\omega$, in view of (3.13), $\left(H_{1}\right)-\left(H_{4}\right)$ and Lemma 2.11, we obtain that

$$
\begin{aligned}
& \int_{0}^{\omega}\left|x_{i}^{\Delta}(t)\right| \Delta t=\sum_{k=1}^{2 q+1} \int_{t_{k-1}^{+}}^{t_{k}}\left|x_{i}^{\Delta}(t)\right| \Delta t+\sum_{k=1}^{2 q}\left|\Delta x_{i}\left(t_{k}\right)\right| \\
& \leq \int_{0}^{\omega}\left|\frac{1}{1+\lambda} G_{i}(t, x)-\frac{\lambda}{1+\lambda} G_{i}(t,-x)\right| \Delta t \\
& +\sum_{k=1}^{2 q}\left|\frac{1}{1+\lambda} I_{i k}\left(x_{i}\left(t_{k}\right)\right)-\frac{\lambda}{1+\lambda} I_{i k}\left(-x_{i}\left(t_{k}\right)\right)\right| \\
& \leq\left[\frac{1}{1+\lambda}+\frac{\lambda}{1+\lambda}\right] \int_{0}^{\omega} \max \left\{\left|G_{i}(t, x)\right|,\left|G_{i}(t,-x)\right|\right\} \Delta t \\
& +\left[\frac{1}{1+\lambda}+\frac{\lambda}{1+\lambda}\right] \sum_{k=1}^{2 q} \max \left\{\left|I_{i k}\left(x_{i}\left(t_{k}\right)\right)\right|,\left|I_{i k}\left(-x_{i}\left(t_{k}\right)\right)\right|\right\} \\
& \leq \int_{0}^{\omega} \max \left\{\left|a_{i}\left(t, x_{i}(t)\right)\left[b_{i}\left(t, x_{i}(t)\right)+f_{i}\left(t, x_{t}\right)\right]\right|\right. \\
& \left.\left|a_{i}\left(t,-x_{i}(t)\right)\left[b_{i}\left(t,-x_{i}(t)\right)+f_{i}\left(t,-x_{t}\right)\right]\right|\right\} \Delta t \\
& +\sum_{k=1}^{2 q} \max \left\{\left|I_{i k}\left(x_{i}\left(t_{k}\right)\right)\right|,\left|I_{i k}\left(-x_{i}\left(t_{k}\right)\right)\right|\right\} \\
& \leq a_{i}^{M}\left[\int_{0}^{\omega} \max \left\{\left|b_{i}\left(t, x_{i}(t)\right)-b_{i}(t, 0)\right|,\left|b_{i}\left(t,-x_{i}(t)\right)-b_{i}(t, 0)\right|\right\} \Delta t\right. \\
& +\int_{0}^{\omega} \max \left\{\left|f_{i}\left(t, x_{1 t}, \ldots, x_{n t}\right)-f_{i}(t, 0, \ldots, 0)\right|,\right. \\
& \left.\left.\left|f_{i}\left(t,-x_{1 t}, \ldots,-x_{n t}\right)-f_{i}(t, 0, \ldots, 0)\right|\right\} \Delta t\right] \\
& +\sum_{k=1}^{2 q} \max \left\{\left|I_{i k}\left(x_{i}\left(t_{k}\right)\right)-I_{i k}(0)\right|,\left|I_{i k}\left(-x_{i}\left(t_{k}\right)\right)-I_{i k}(0)\right|\right\}+\sum_{k=1}^{2 q}\left|I_{i k}(0)\right| \\
& \leq a_{i}^{M}\left[L_{i}^{b} \int_{0}^{\omega}\left|x_{i}(t)\right| \Delta t+\int_{0}^{\omega} c_{i} \sum_{j=1}^{n}\left|x_{j t}\right| \Delta t\right]+\sum_{k=1}^{2 q} L_{i k}^{I}\left|x_{i}\right|_{0}+\sum_{k=1}^{2 q}\left|I_{i k}(0)\right| \\
& \leq a_{i}^{M} L_{i}^{b} \sqrt{\omega}\left\|x_{i}\right\|_{2}+a_{i}^{M} c_{i} \sum_{j=1}^{n}\left\|x_{j}\right\|_{2} \sqrt{\omega}+\sum_{k=1}^{2 q} L_{i k}^{I}\left|x_{i}\right|_{0}+\sum_{k=1}^{2 q}\left|I_{i k}(0)\right|,
\end{aligned}
$$

where $i=1,2, \ldots, n$. Integrating (3.13) from 0 to $\omega$, we have from $\left(H_{1}\right)-\left(H_{4}\right)$ that

$$
\left|\int_{0}^{\omega}\left[\frac{a_{i}\left(t, x_{i}(t)\right) b_{i}\left(t, x_{i}(t)\right)}{1+\lambda}-\frac{\lambda a_{i}\left(t,-x_{i}(t)\right) b_{i}\left(t,-x_{i}(t)\right)}{1+\lambda}\right] \Delta t\right|
$$




$$
\begin{aligned}
= & \left|\int_{0}^{\omega}\left[\frac{a_{i}\left(t, x_{i}(t)\right) b_{i}\left(t, x_{i}(t)\right)}{1+\lambda}+\frac{\lambda a_{i}\left(t, x_{i}(t)\right) b_{i}\left(t, x_{i}(t)\right)}{1+\lambda}\right] \Delta t\right| \\
= & \left|\int_{0}^{\omega} a_{i}\left(t, x_{i}(t)\right) b_{i}\left(t, x_{i}(t)\right) \Delta t\right| \\
= & \mid \frac{1}{1+\lambda} \int_{0}^{\omega} a_{i}\left(t, x_{i}(t)\right) f_{i}\left(t, x_{t}\right) \Delta t-\frac{\lambda}{1+\lambda} \int_{0}^{\omega} a_{i}\left(t,-x_{i}(t)\right) f_{i}\left(t,-x_{t}\right) \Delta t \\
& +\frac{1}{1+\lambda} \sum_{k=1}^{2 q} I_{i k}\left(x_{i}\left(t_{k}\right)\right)-\frac{\lambda}{1+\lambda} \sum_{k=1}^{2 q} I_{i k}\left(-x_{i}\left(t_{k}\right)\right) \mid \\
\leq & a_{i}^{M} \int_{0}^{\omega} \max \left\{\left|f_{i}\left(t, x_{1 t}, \ldots, x_{n t}\right)-\right| f_{i}(t, 0, \ldots, 0) \mid,\right. \\
& +\sum_{k=1}^{2 q} \max \left\{\left|I_{i k}\left(x_{i}\left(t,-x_{k}\right)\right)-I_{i k}(0)\right|,\left|I_{i k}\left(-x_{i}\left(t_{k}\right)\right)-I_{i k}(0)\right|\right\}+\sum_{k=1}^{2 q}\left|I_{i k}(0)\right| \\
\leq & a_{i}^{M} c_{i} \sum_{j=1}^{n}\left\|x_{j}\right\|_{2} \sqrt{\omega}+\sum_{k=1}^{2 q} L_{i k}^{I}\left|x_{i}\right|_{0}+\sum_{k=1}^{2 q}\left|I_{i k}(0)\right|, \quad i=1,2, \ldots, n,
\end{aligned}
$$

by $\left(H_{2}\right)$, we obtain that

$$
\left|\int_{0}^{\omega} a_{i}\left(t, x_{i}(t)\right) x_{i}(t) \Delta t\right| \leq \frac{1}{\mu_{i}} a_{i}^{M} c_{i} \sum_{j=1}^{n}\left\|x_{j}\right\|_{2} \sqrt{\omega}+\frac{1}{\mu_{i}} \sum_{k=1}^{2 q} L_{i k}^{I}\left|x_{i}\right|_{0}+\frac{1}{\mu_{i}} \sum_{k=1}^{2 q}\left|I_{i k}(0)\right|,
$$

where $i=1,2, \ldots, n$. From Lemma 2.10, for any $t_{1}^{i}, t_{2}^{i} \in[0, \omega]_{\mathbb{T}}, i=1,2, \ldots, n$, we have

$$
\begin{aligned}
& \int_{0}^{\omega} a_{i}\left(t, x_{i}(t)\right) x_{i}(t) \Delta t \leq \int_{0}^{\omega} a_{i}\left(t, x_{i}(t)\right) x_{i}\left(t_{1}^{i}\right) \Delta t+\int_{0}^{\omega} a_{i}\left(t, x_{i}(t)\right)\left(\int_{0}^{\omega}\left|x_{i}^{\Delta}(t)\right| \Delta t\right) \Delta t, \\
& \int_{0}^{\omega} a_{i}\left(t, x_{i}(t)\right) x_{i}(t) \Delta t \geq \int_{0}^{\omega} a_{i}\left(t, x_{i}(t)\right) x_{i}\left(t_{2}^{i}\right) \Delta t-\int_{0}^{\omega} a_{i}\left(t, x_{i}(t)\right)\left(\int_{0}^{\omega}\left|x_{i}^{\Delta}(t)\right| \Delta t\right) \Delta t .
\end{aligned}
$$

Dividing by $\int_{0}^{\omega} a_{i}\left(t, x_{i}(t)\right) \Delta t$ on the both sides of (3.18) and (3.19), respectively, we obtain that

$$
\begin{aligned}
& x_{i}\left(t_{1}^{i}\right) \geq \frac{1}{\int_{0}^{\omega} a_{i}\left(t, x_{i}(t)\right) \Delta t} \int_{0}^{\omega} a_{i}\left(t, x_{i}(t)\right) x_{i}(t) \Delta t-\int_{0}^{\omega}\left|x_{i}^{\Delta}(t)\right| \Delta t, \quad i=1,2, \ldots, n, \\
& x_{i}\left(t_{2}^{i}\right) \leq \frac{1}{\int_{0}^{\omega} a_{i}\left(t, x_{i}(t)\right) \Delta t} \int_{0}^{\omega} a_{i}\left(t, x_{i}(t)\right) x_{i}(t) \Delta t+\int_{0}^{\omega}\left|x_{i}^{\Delta}(t)\right| \Delta t, \quad i=1,2, \ldots, n .
\end{aligned}
$$


Let $\bar{t}_{i}, \underline{t}_{i} \in[0, \omega]_{\mathbb{T}}$, such that $x_{i}\left(\bar{t}_{i}\right)=\max _{t \in[0, \omega]_{\mathbb{T}}} x_{i}(t), x_{i}\left(\underline{t}_{i}\right)=\min _{t \in[0, \omega]_{\mathbb{T}}} x_{i}(t)$, by the arbitrariness of $t_{1}^{i}, t_{2}^{i}$ in view of (3.15), (3.17), (3.20), we have

$$
\begin{aligned}
x_{i}\left(t_{1}^{i}\right) \geq & \frac{1}{\int_{0}^{\omega} a_{i}\left(t, x_{i}(t)\right) \Delta t} \int_{0}^{\omega} a_{i}\left(t, x_{i}(t)\right) x_{i}(t) \Delta t-\int_{0}^{\omega}\left|x_{i}^{\Delta}(t)\right| \Delta t \\
\geq & -\frac{1}{\int_{0}^{\omega} a_{i}\left(t, x_{i}(t)\right) \Delta t}\left|\int_{0}^{\omega} a_{i}\left(t, x_{i}(t)\right) x_{i}(t) \Delta t\right|-\int_{0}^{\omega}\left|x_{i}^{\Delta}(t)\right| \Delta t \\
\geq & -\frac{1}{\omega a_{i}^{m}}\left[\frac{1}{\mu_{i}} a_{i}^{M} \sum_{j=1}^{n} c_{i}\left\|x_{j}\right\|_{2} \sqrt{\omega}+\frac{1}{\mu_{i}} \sum_{k=1}^{2 q} L_{i k}^{I}\left|x_{i}\right|_{0}+\frac{1}{\mu_{i}} \sum_{k=1}^{2 q}\left|I_{i k}(0)\right|\right] \\
& -\left[a_{i}^{M} L_{i}^{b} \sqrt{\omega}\left\|x_{j}\right\|_{2}+a_{i}^{M} c_{i} \sum_{j=1}^{n}\left\|x_{j}\right\|_{2} \sqrt{\omega}+\sum_{k=1}^{2 q} L_{i k}^{I}\left|x_{i}\right|_{0}+\sum_{k=1}^{2 q}\left|I_{i k}(0)\right|\right], \\
x_{i}\left(t_{2}^{i}\right) \leq & \frac{1}{\int_{0}^{\omega} a_{i}\left(t, x_{i}(t)\right) \Delta t} \int_{0}^{\omega} a_{i}\left(t, x_{i}(t)\right) x_{i}(t) \Delta t+\int_{0}^{\omega}\left|x_{i}^{\Delta}(t)\right| \Delta t \\
\leq & \frac{1}{\int_{0}^{\omega} a_{i}\left(t, x_{i}(t)\right) \Delta t}\left|\int_{0}^{\omega} a_{i}\left(t, x_{i}(t)\right) x_{i}(t) \Delta t\right|+\int_{0}^{\omega}\left|x_{i}^{\Delta}(t)\right| \Delta t \\
\leq & \frac{1}{\omega a_{i}^{m}}\left[\frac{1}{\mu_{i}} a_{i}^{M} c_{i} \sum_{j=1}^{n}\left\|x_{j}\right\|_{2} \sqrt{\omega}+\frac{1}{\mu_{i}} \sum_{k=1}^{2 q} L_{i k}^{I}\left|x_{i}\right|_{0}+\frac{1}{\mu_{i}} \sum_{k=1}^{2 q}\left|I_{i k}(0)\right|\right] \\
& +\left[a_{i}^{M} L_{i}^{b} \sqrt{\omega}\left\|x_{j}\right\|_{2}+a_{i}^{M} c_{i} \sum_{j=1}^{n}\left\|x_{j}\right\|_{2} \sqrt{\omega}+\sum_{k=1}^{2 q} L_{i k}^{I}\left|x_{i}\right|_{0}+\sum_{k=1}^{2 q}\left|I_{i k}(0)\right|\right]
\end{aligned}
$$

where $i=1,2, \ldots, n$. Thus, we have from (3.21) that

$$
\begin{aligned}
\left|x_{i}\right|_{0}= & \max _{t \in[0, \omega]_{\mathbb{T}}}\left|x_{i}(t)\right| \\
\leq & \frac{1}{\omega a_{i}^{m}}\left[\frac{1}{\mu_{i}} a_{i}^{M} c_{i} \sum_{j=1}^{n}\left\|x_{j}\right\|_{2} \sqrt{\omega}+\frac{1}{\mu_{i}} \sum_{k=1}^{2 q} L_{i k}^{I}\left|x_{i}\right|_{0}+\frac{1}{\mu_{i}} \sum_{k=1}^{2 q}\left|I_{i k}(0)\right|\right] \\
& +\left[a_{i}^{M} L_{i}^{b} \sqrt{\omega}\left\|x_{i}\right\|_{2}+a_{i}^{M} c_{i} \sum_{j=1}^{n}\left\|x_{j}\right\|_{2} \sqrt{\omega}+\sum_{k=1}^{2 q} L_{i k}^{I}\left|x_{i}\right|_{0}+\sum_{k=1}^{2 q}\left|I_{i k}(0)\right|\right],
\end{aligned}
$$

where $i=1,2, \ldots, n$. In addition, we have that

$$
\left\|x_{i}\right\|_{2}=\left(\int_{0}^{\omega}\left|x_{i}(s)\right| \Delta s\right)^{1 / 2} \leq \sqrt{\omega} \max _{t \in[0, \omega]_{\mathbb{T}}}\left|x_{i}(t)\right|=\sqrt{\omega}\left|x_{i}\right|_{0}, \quad i=1,2, \ldots, n .
$$


By (3.22), we obtain that,

$$
\begin{aligned}
\omega a_{i}^{m}\left|x_{i}\right|_{0} \leq & {\left[\frac{1}{\mu_{i}} a_{i}^{M} c_{i} \sum_{j=1}^{n}\left\|x_{j}\right\|_{2} \sqrt{\omega}+\frac{1}{\mu_{i}} \sum_{k=1}^{2 q} L_{i k}^{I}\left|x_{i}\right|_{0}+\frac{1}{\mu_{i}} \sum_{k=1}^{2 q}\left|I_{i k}(0)\right|\right] } \\
& +\omega a_{i}^{m}\left[a_{i}^{M} L_{i}^{b} \sqrt{\omega}\left\|x_{i}\right\|_{2}+a_{i}^{M} c_{i} \sum_{j=1}^{n}\left\|x_{j}\right\|_{2} \sqrt{\omega}+\sum_{k=1}^{2 q} L_{i k}^{I}\left|x_{i}\right|_{0}+\sum_{k=1}^{2 q}\left|I_{i k}(0)\right|\right] \\
\leq & {\left[\frac{1}{\mu_{i}} a_{i}^{M} \omega c_{i} \sum_{j=1}^{n}\left|x_{j}\right|_{0}+\frac{1}{\mu_{i}} \sum_{k=1}^{2 q} \mathrm{~L}_{i k}^{I}\left|x_{i}\right|_{0}+\frac{1}{\mu_{i}} \sum_{k=1}^{2 q}\left|I_{i k}(0)\right|\right] } \\
& +\omega a_{i}^{m}\left[a_{i}^{M} L_{i}^{b} \omega\left|x_{i}\right|_{0}+a_{i}^{M} \omega c_{i} \sum_{j=1}^{n}\left|x_{j}\right|_{0}+\sum_{k=1}^{2 q} L_{i k}^{I}\left|x_{i}\right|_{0}+\sum_{k=1}^{2 q}\left|I_{i k}(0)\right|\right]
\end{aligned}
$$

where $i=1,2, \ldots, n$. That is,

$$
\begin{gathered}
{\left[\omega a_{i}^{m}-\omega^{2} a_{i}^{m} a_{i}^{M} L_{i}^{b}-\omega a_{i}^{m} \sum_{k=1}^{2 q} L_{i k}^{I}-\frac{1}{\mu_{i}} \sum_{k=1}^{2 q} L_{i k}^{I}\right]\left|x_{i}\right|_{0}-\left[\left(\frac{1}{\mu_{i}}+\omega a_{i}^{m}\right) a_{i}^{M} \omega c_{i}\right]\left|x_{j}\right|_{0}} \\
\leq \frac{1}{\mu_{i}} \sum_{k=1}^{2 q}\left|I_{i k}(0)\right|+\omega a_{i}^{m} \sum_{k=1}^{2 q}\left|I_{i k}(0)\right|=D_{i}, \quad i=1,2, \ldots, n .
\end{gathered}
$$

Denote that,

$$
|x|_{0}=\left(\left|x_{1}\right|_{0},\left|x_{2}\right|_{0}, \ldots,\left|x_{n}\right|_{0}\right)^{T}, \quad D=\left(D_{1}, D_{2}, \ldots, D_{n}\right)^{T} .
$$

Then (3.25) can be rewritten in the matrix form

$$
E|x|_{0} \leq D
$$

From the conditions of Theorem 3.1, $E$ is a nonsingular $M$ matrix, therefore,

$$
|x|_{0} \leq E^{-1} D \triangleq\left(M_{1}, M_{2}, \ldots, M_{n}\right)^{T} .
$$

Let

$$
M=\sum_{i=1}^{n} M_{i}+1 \quad(\text { Clearly, } M \text { is independent of } \lambda)
$$

Take

$$
\Omega=\left\{x \in \mathbb{X}:\|x\|_{\mathbb{X}}<M\right\}
$$


It is clear that $\Omega$ satisfies all the requirements in Lemma 2.13 and condition $(H)$ is satisfied. In view of all the discussions above, we conclude from Lemma 2.13 that system (1.1) has at least one $\omega / 2$-anti-periodic solution. This completes the proof.

\section{Global Exponential Stability of Antiperiodic Solution}

Suppose that $x^{*}(t)=\left(x_{1}^{*}(t), x_{2}^{*}(t), \ldots, x_{n}^{*}(t)\right)^{T}$ is an $\omega / 2$-anti-periodic solution of system (1.1). In this section, we will construct some suitable Lyapunov functions to study the global exponential stability of this anti-periodic solution.

Theorem 4.1. Assume that $\left(H_{1}\right)-\left(H_{5}\right)$ hold. Suppose further that

$\left(H_{6}\right)$ there exist positive constants $L_{i}^{a}$ such that

$$
\left|a_{i}(t, u)-a_{i}(t, v)\right| \leq L_{i}^{a}|u-v|, \quad \forall u, v \in \mathbb{R}, \quad i=1,2, \ldots, n
$$

$\left(H_{7}\right)$ for all $u, v \in \mathbb{R}, i=1,2, \ldots, n$, there exist positive constants $L_{i}^{a b}$ such that

$$
\begin{aligned}
& {\left[a_{i}(t, u) b_{i}(t, u)-a_{i}(t, v) b_{i}(t, v)\right](u-v) \leq 0, \quad i=1,2, \ldots, n,} \\
& \left|a_{i}(t, u) b_{i}(t, u)-a_{i}(t, v) b_{i}(t, v)\right| \geq L_{i}^{a b}|u-v|, \quad i=1,2, \ldots, n
\end{aligned}
$$

$\left(H_{8}\right)$ there are $\omega$-periodic functions $r_{i}(t)$ such that $r_{i}(t)=\sup _{u \in \mathbb{R}}\left|f_{i}(t, u)\right|, i=1,2, \ldots, n$;

$\left(H_{9}\right)$ there exists a positive constant $\epsilon$ such that

$$
\begin{aligned}
\Psi_{i}(\epsilon, t)= & {\left[\epsilon+(1+\epsilon \mu(t))\left(-L_{i}^{a b}+L_{i}^{a} r_{i}^{M}\right)\right] } \\
& +\sum_{j=1}^{n}(1+\epsilon \mu(t-\theta)) e_{\epsilon}(t-\theta, t) a_{i}^{M} c_{i}>0, \quad i=1,2, \ldots, n
\end{aligned}
$$

$\left(H_{10}\right)$ impulsive operator $I_{i k}\left(x_{i}\left(t_{k}\right)\right.$ satisfy

$$
I_{i k}\left(x_{i}\left(t_{k}\right)\right)=-\gamma_{i k}\left(x_{i}\left(t_{k}\right)\right), \quad 0<\gamma_{i k}<2, \quad i=1, \ldots, n, \quad k \in \mathbb{N} .
$$

Then the $\omega / 2$-anti-periodic solution of system (1.1) is globally exponentially stable.

Proof. According to Theorem 3.1, we know that system (1.1) has an $\omega / 2$-anti-periodic solution $x^{*}(t)=\left(x_{1}^{*}(t), x_{2}^{*}(t), \ldots, \mathrm{x}_{n}^{*}(t)\right)^{T}$ with initial value $x^{*}(s), s \in E_{\mathbb{T}}$, suppose that 
$x(t)=\left(x_{1}(t), x_{2}(t), \ldots, x_{n}(t)\right)^{T}$ is an arbitrary solution of system (1.1) with initial value $\phi(s), s \in E_{\mathbb{T}}$. Then it follows from system (1.1) that

$$
\begin{aligned}
\left(x_{i}(t)-x_{i}^{*}(t)\right)^{\Delta}= & a_{i}\left(t, x_{i}(t)\right) b_{i}\left(t, x_{i}(t)\right)-a_{i}\left(t, x_{i}^{*}(t)\right) b_{i}\left(t, x_{i}^{*}(t)\right)+a_{i}\left(t, x_{i}(t)\right) f_{i}\left(t, x_{t}\right) \\
& -a_{i}\left(t, x_{i}^{*}(t)\right) f_{i}\left(t, x_{t}^{*}\right), \quad t \in \mathbb{T}^{+}, \quad t \neq t_{k}, \\
\Delta\left(x_{i}\left(t_{k}\right)-x_{i}^{*}\left(t_{k}\right)\right)= & -\gamma_{i k}\left(x_{i}\left(t_{k}\right)-x_{i}^{*}\left(t_{k}\right)\right), \quad t=t_{k}, \quad k \in \mathbb{N}, \quad i=1,2, \ldots, n .
\end{aligned}
$$

In view of system (4.5), for $t \in \mathbb{T}^{+}, t \neq t_{k}, k \in \mathbb{N}, i=1,2, \ldots, n$, we have

$$
\begin{aligned}
\left(x_{i}(t)-x_{i}^{*}(t)\right)^{\Delta}= & a_{i}\left(t, x_{i}(t)\right) b_{i}\left(t, x_{i}(t)\right)-a_{i}\left(t, x_{i}^{*}(t)\right) b_{i}\left(t, x_{i}^{*}(t)\right) \\
& +a_{i}\left(t, x_{i}(t)\right) f_{i}\left(t, x_{t}\right)-a_{i}\left(t, x_{i}^{*}(t)\right) f_{i}\left(t, x_{t}^{*}\right) \\
= & {\left[a_{i}\left(t, x_{i}(t)\right) b_{i}\left(t, x_{i}(t)\right)-a_{i}\left(t, x_{i}^{*}(t)\right) b_{i}\left(t, x_{i}^{*}(t)\right)\right] } \\
& +\left[a_{i}\left(t, x_{i}(t)\right)-a_{i}\left(t, x_{i}^{*}(t)\right)\right] f_{i}\left(t, x_{t}\right)+a_{i}\left(t, x_{i}^{*}(t)\right)\left[f_{i}\left(t, x_{t}\right)-f_{i}\left(t, x_{t}^{*}\right)\right] .
\end{aligned}
$$

Hence, we can obtain from $\left(H_{6}\right)-\left(H_{9}\right)$ that

$$
\begin{aligned}
D^{+}\left|x_{i}(t)-x_{i}^{*}(t)\right|^{\Delta} \leq & -L_{i}^{a b}\left|x_{i}(t)-x_{i}^{*}(t)\right|+L_{i}^{a} r_{i}^{M}\left|x_{i}(t)-x_{i}^{*}(t)\right| \\
& +a_{i}^{M} \sum_{j=1}^{n} c_{i}\left|x_{j}(t+\theta)-x_{j}^{*}(t+\theta)\right| \\
= & \left(-L_{i}^{a b}+L_{i}^{a} r_{i}^{M}\right)\left|x_{i}(t)-x_{i}^{*}(t)\right|+a_{i}^{M} c_{i} \sum_{j=1}^{n}\left|x_{j}(t+\theta)-x_{j}^{*}(t+\theta)\right|,
\end{aligned}
$$

for $i=1,2, \ldots, n$, and we have from $\left(H_{10}\right)$ that

$$
\left|x_{i}\left(t_{k}^{+}\right)-x_{i}^{*}\left(t_{k}^{+}\right)\right|=\left|1-\gamma_{i k}\right|\left|x_{i}\left(t_{k}\right)-x_{i}^{*}\left(t_{k}\right)\right|, \quad i=1,2, \ldots, n, \quad k \in \mathbb{N} .
$$

For any $\alpha \in E$, we construct the Lyapunov functional

$$
\begin{aligned}
V(t) & =V_{1}(t)+V_{2}(t), \\
V_{1}(t) & =\sum_{i=1}^{n} e_{\epsilon}(t, \alpha)\left|x_{i}(t)-x_{i}^{*}(t)\right|, \\
V_{2}(t) & =\sum_{i=1}^{n} \sum_{j=1}^{n} \int_{t+\theta}^{t}(1+\epsilon \mu(s-\theta)) e_{\epsilon}(s-\theta, \alpha) a_{i}^{M} c_{i}\left|x_{j}(s)-x_{j}^{*}(s)\right| \Delta s .
\end{aligned}
$$


For $t \in \mathbb{T}^{+}, t \neq t_{k}, k \in \mathbb{N}$, calculating the delta derivative $D^{+} V(t)^{\Delta}$ of $V(t)$ along solutions of system (4.5), we can get

$$
\begin{aligned}
D^{+} V_{1}(t)^{\Delta} \leq & \sum_{i=1}^{n} \epsilon e_{\epsilon}(t, \alpha)\left|x_{i}(t)-x_{i}^{*}(t)\right|+\sum_{i=1}^{n} e_{\epsilon}(\sigma(t), \alpha) D^{+}\left|x_{i}(t)-x_{i}^{*}(t)\right|^{\Delta} \\
\leq & \sum_{i=1}^{n}\left\{\epsilon e_{\epsilon}(t, \alpha)\left|x_{i}(t)-x_{i}^{*}(t)\right|+e_{\epsilon}(\sigma(t), \alpha)\right. \\
& \left.\quad \times\left[\left(-L_{i}^{a b}+L_{i}^{a} r_{i}^{M}\right)\left|x_{i}(t)-x_{i}^{*}(t)\right|+a_{i}^{M} c_{i} \sum_{j=1}^{n}\left|x_{j}(t+\theta)-x_{j}^{*}(t+\theta)\right|\right]\right\} \\
= & \sum_{i=1}^{n}\left[\epsilon+(1+\epsilon \mu(t))\left(-L_{i}^{a b}+L_{i}^{a} r_{i}^{M}\right)\right] e_{\epsilon}(t, \alpha)\left|x_{i}(t)-x_{i}^{*}(t)\right| \\
& +(1+\epsilon \mu(t)) e_{\epsilon}(t, \alpha) c_{i} \sum_{i=1}^{n} \sum_{j=1}^{n} a_{i}^{M}\left|x_{j}(t+\theta)-x_{j}^{*}(t+\theta)\right| \\
D^{+} V_{2}(t)^{\Delta} \leq & \sum_{i=1}^{n} \sum_{j=1}^{n}(1+\epsilon \mu(t-\theta)) e_{\epsilon}(t-\theta, \alpha) a_{i}^{M} c_{i}\left|x_{j}(t)-x_{j}^{*}(t)\right| \\
& \quad-\sum_{i=1}^{n} \sum_{j=1}^{n}(1+\epsilon \mu(t)) e_{\epsilon}(t, \alpha) a_{i}^{M} c_{i}\left|x_{j}(t+\theta)-x_{j}^{*}(t+\theta)\right| .
\end{aligned}
$$

By assumption $\left(H_{8}\right)$, it concludes that

$$
\begin{aligned}
D^{+} V(t)^{\Delta}= & D^{+} V_{1}(t)^{\Delta}+D^{+} V_{2}(t)^{\Delta} \\
\leq & \sum_{i=1}^{n}\left[\epsilon+(1+\epsilon \mu(t))\left(-L_{i}^{a b}+L_{i}^{a} r_{i}^{M}\right)\right] e_{\epsilon}(t, \alpha)\left|x_{i}(t)-x_{i}^{*}(t)\right| \\
& +\sum_{i=1}^{n} \sum_{j=1}^{n}(1+\epsilon \mu(t-\theta)) e_{\epsilon}(t-\theta, \alpha) a_{i}^{M} c_{i}\left|x_{j}(t)-x_{j}^{*}(t)\right| \\
\leq & \sum_{i=1}^{n}\left\{\left[\epsilon+(1+\epsilon \mu(t))\left(-L_{i}^{a b}+L_{i}^{a} r_{i}^{M}\right)\right]\right. \\
& \left.\quad+\sum_{j=1}^{n}(1+\epsilon \mu(t-\theta)) e_{\epsilon}(t-\theta, t) a_{i}^{M} c_{i}\right\} e_{\epsilon}(t, \alpha)\left|x_{i}(t)-x_{i}^{*}(t)\right| \\
\leq & 0, \quad t \in \mathbb{T}^{+}, \quad t \neq t_{k}, \quad k \in \mathbb{N} .
\end{aligned}
$$


Also,

$$
\begin{aligned}
V\left(t_{k}^{+}\right)= & V_{1}\left(t_{k}^{+}\right)+V_{2}\left(t_{k}^{+}\right) \\
= & \sum_{i=1}^{n} e_{\epsilon}\left(t_{k^{\prime}}^{+} \alpha\right)\left|x_{i}\left(t_{k}^{+}\right)-x_{i}^{*}\left(t_{k}^{+}\right)\right| \\
& +\sum_{i=1}^{n} \sum_{j=1}^{n} \int_{t_{k}^{+}+\theta}^{t_{k}^{+}}(1+\epsilon \mu(s-\theta)) e_{\epsilon}(s-\theta, \alpha) a_{i}^{M} c_{i}\left|x_{j}(s)-x_{j}^{*}(s)\right| \Delta s \\
\leq & \sum_{i=1}^{n} e_{\epsilon}\left(t_{k}, \alpha\right)\left|x_{i}\left(t_{k}\right)-x_{i}^{*}\left(t_{k}\right)\right| \\
& +\sum_{i=1}^{n} \sum_{j=1}^{n} \int_{t_{k}+\theta}^{t_{k}}(1+\epsilon \mu(s-\theta)) e_{\epsilon}(s-\theta, \alpha) a_{i}^{M} c_{i}\left|x_{j}(s)-x_{j}^{*}(s)\right| \Delta s \\
= & V\left(t_{k}\right), \quad k \in \mathbb{N} .
\end{aligned}
$$

It follows that $V(t) \leq V(0)$ for all $t \in \mathbb{T}^{+}$.

On the other hand, we have

$$
\begin{aligned}
V(0)= & V_{1}(0)+V_{2}(0) \\
= & \sum_{i=1}^{n} e_{\epsilon}(0, \alpha)\left|x_{i}(0)-x_{i}^{*}(0)\right| \\
& +\sum_{i=1}^{n} \sum_{j=1}^{n} \int_{\theta}^{0}(1+\epsilon \mu(s-\theta)) e_{\epsilon}(s-\theta, \alpha) a_{i}^{M} c_{i}\left|x_{j}(s)-x_{j}^{*}(s)\right| \Delta s \\
\leq & \sum_{i=1}^{n}\left\{e_{\epsilon}(0, \alpha)+\sum_{j=1}^{n} \int_{\theta}^{0}(1+\epsilon \mu(s-\theta)) e_{\epsilon}(s-\theta, \alpha) a_{i}^{M} c_{i} \Delta s\right\} \sup _{s \in E_{\mathbb{T}}}\left|x_{i}(s)-x_{i}^{*}(s)\right| \\
\leq & M(\epsilon) \sum_{i=1}^{n} \sup _{s \in E_{\mathbb{T}}}\left|\phi_{i}(s)-x_{i}^{*}(s)\right|,
\end{aligned}
$$

where

$$
M(\epsilon)=\max _{1 \leq i \leq n}\left\{\sup _{\alpha \in E_{\mathbb{T}}}\left(e_{\epsilon}(0, \alpha)+\sum_{j=1}^{n} \int_{\theta}^{0}(1+\epsilon \mu(s-\theta)) e_{\epsilon}(s-\theta, \alpha) a_{i}^{M} c_{i j} \Delta s\right)\right\} .
$$

It is obvious that

$$
\sum_{i=1}^{n} e_{\epsilon}(0, \alpha)\left|x_{i}(t)-x_{i}^{*}(t)\right| \leq V(t) \leq V(0) \leq M(\epsilon) \sup _{s \in E_{\mathbb{T}}} \sum_{i=1}^{n}\left|\phi_{i}(s)-x_{i}^{*}(s)\right|
$$


So we can finally get

$$
\sum_{i=1}^{n}\left|x_{i}(t)-x_{i}^{*}(t)\right| \leq M(\epsilon) e_{\ominus \epsilon}(0, \alpha) \sup _{s \in E_{\mathbb{T}}} \sum_{i=1}^{n}\left|\phi_{i}(s)-x_{i}^{*}(s)\right|=M(\epsilon) e_{\ominus \epsilon}(0, \alpha)\left\|\phi-x^{*}\right\| .
$$

Since $M(\epsilon) \geq 1$, from Definition 2.12, the $\omega / 2$-anti-periodic solution of system (1.1) is globally exponential stable. This completes the proof.

\section{An Example}

Example 5.1. Consider the following impulsive generalized neural networks:

$$
\begin{aligned}
x^{\Delta}(t) & =A(t, x(t))\left[B(t, x(t))+F\left(t, x_{t}\right)\right], \quad t \in \mathbb{T}, \quad t \neq t_{k}, \\
\Delta x\left(t_{k}\right) & =x\left(t_{k}^{+}\right)-x\left(t_{k}^{-}\right)=I_{k}\left(x\left(t_{k}\right)\right), \quad t=t_{k}, \quad k \in \mathbb{Z},
\end{aligned}
$$

where

$$
\begin{gathered}
A(t, u)=\operatorname{diag}\left\{10+\frac{2}{\pi} \arctan |u|, 11+\frac{2}{\pi} \arctan |u|\right\} \\
B(t, u)=\frac{1}{100}\left(\begin{array}{l}
u \\
u
\end{array}\right), F\left(t, x_{t}\right)=\left(\begin{array}{c}
\sum_{j=1}^{2} c_{i}(t) g_{j}\left(x_{j t}\right) \\
\sum_{j=1}^{2} c_{i}(t) g_{j}\left(x_{j t}\right)
\end{array}\right), \\
\left(g_{j}\right)_{2 \times 1}=\frac{1}{1000}\left(\begin{array}{c}
\sin u \\
\sin u
\end{array}\right), \quad\left(c_{i}\right)_{2 \times 1}=\frac{1}{1000}\left(\begin{array}{c}
\sin t \\
\cos t
\end{array}\right), \quad\left(I_{k}\right)_{2 \times 2}=\frac{1}{500}\left(\begin{array}{ll}
-u & -u \\
-u & -u
\end{array}\right), \\
\omega=2 \pi, \quad[0,2 \pi]_{\mathbb{T}} \cap\left\{t_{k}: k \in \mathbb{N}\right\}=\left\{t_{1}, t_{2}\right\},
\end{gathered}
$$

when $\mathbb{T}=\mathbb{R}$, system (5.1) has at least one exponentially stable $\pi$-anti-periodic solution.

Proof. By calculation, we have $a_{1}^{m}=10, a_{1}^{M}=11, a_{2}^{m}=11, a_{2}^{m}=12, L_{1}^{a}=L_{2}^{a}=2 / \pi, L_{1}^{b}=L_{2}^{b}=$ $1 / 100, c_{1}^{M}=1 / 1000, c_{2}^{M}=1 / 1000, L_{11}^{I}=L_{21}^{I}=L_{12}^{I}=L_{22}^{I}=1 / 500$, and $\mu_{1}=\mu_{2}=1 / 100$. It is obvious that $\left(H_{1}\right)-\left(H_{4}\right),\left(H_{6}\right)-\left(H_{8}\right)$, and $\left(H_{10}\right)$ are satisfied. Furthermore, we can easily calculate that

$$
E \approx\left(\begin{array}{cc}
7.52 & -12.74 \\
-11.25 & 3.61
\end{array}\right)
$$

is a nonsingular $M$ matrix, thus $\left(H_{5}\right)$ is satisfied.

When $\mathbb{T}=\mathbb{R}, \mu(t)=0$. Take $\epsilon=0.01, \theta=-1$, we have that

$$
\Psi_{1}(\epsilon, t) \approx-0.04<0, \quad \Psi_{2}(\epsilon, t) \approx-0.03<0
$$


Hence $\left(H_{10}\right)$ holds. By Theorems 3.1 and 4.1 , system (5.1) has at least one exponentially stable $\pi$-anti-periodic solution. This completes the proof.

\section{Conclusions}

Using the time scales calculus theory, the coincidence degree theory, and the Lyapunov functional method, we obtain sufficient conditions for the existence and global exponential stability of anti-periodic solutions for a class of generalized neural networks with impulses and arbitrary delays. This class of generalized neural networks include many continuous or discrete time neural networks such as, Hopfield type neural networks, cellular neural networks, Cohen-Grossberg neural networks, and so on. To the best of our knowledge, the known results about the existence of anti-periodic solutions for neural networks are all done by a similar analytic method, and only good for neural networks without impulse. Our results obtained in this paper are completely new even if the time scale $\mathbb{T}=\mathbb{R}$ or $\mathbb{Z}$ and are of great significance in designs and applications of globally stable anti-periodic Cohen-Grossberg neural networks with delays and impulses .

\section{Acknowledgment}

This work is supported by the National Natural Sciences Foundation of China under Grant 10971183.

\section{References}

[1] X. Li, "Existence and global exponential stability of periodic solution for impulsive CohenGrossberg-type BAM neural networks with continuously distributed delays," Applied Mathematics and Computation, vol. 215, no. 1, pp. 292-307, 2009.

[2] C. Bai, "Global exponential stability and existence of periodic solution of Cohen-Grossberg type neural networks with delays and impulses," Nonlinear Analysis, vol. 9, no. 3, pp. 747-761, 2008.

[3] Z. Chen, D. Zhao, and X. Fu, "Discrete analogue of high-order periodic Cohen-Grossberg neural networks with delay," Applied Mathematics and Computation, vol. 214, no. 1, pp. 210-217, 2009.

[4] Y. K. Li, "Global stability and existence of periodic solutions of discrete delayed cellular neural networks," Physics Letters. A, vol. 333, no. 1-2, pp. 51-61, 2004.

[5] Y. K. Li and Z. Xing, "Existence and global exponential stability of periodic solution of CNNs with impulses," Chaos, Solitons and Fractals, vol. 33, no. 5, pp. 1686-1693, 2007.

[6] Y. K. Li and L. Lu, "Global exponential stability and existence of periodic solution of Hopfield-type neural networks with impulses," Physics Letters. A, vol. 333, no. 1-2, pp. 62-71, 2004.

[7] Z. Zhang and D. Zhou, "Global robust exponential stability for second-order Cohen-Grossberg neural networks with multiple delays," Neurocomputing, vol. 73, no. 1-3, pp. 213-218, 2009.

[8] J. Zhang and Z. Gui, "Existence and stability of periodic solutions of high-order Hopfield neural networks with impulses and delays," Journal of Computational and Applied Mathematics, vol. 224, no. 2, pp. 602-613, 2009.

[9] K. Li, "Stability analysis for impulsive Cohen-Grossberg neural networks with time-varying delays and distributed delays," Nonlinear Analysis, vol. 10, no. 5, pp. 2784-2798, 2009.

[10] H. Okochi, "On the existence of periodic solutions to nonlinear abstract parabolic equations," Journal of the Mathematical Society of Japan, vol. 40, no. 3, pp. 541-553, 1988.

[11] H. Okochi, "On the existence of anti-periodic solutions to nonlinear parabolic equations in noncylindrical domains," Nonlinear Analysis, vol. 14, no. 9, pp. 771-783, 1990.

[12] Y. Q. Chen, "On Massera's theorem for anti-periodic solution," Advances in Mathematical Sciences and Applications, vol. 9, no. 1, pp. 125-128, 1999. 
[13] Y. Yin, "Monotone iterative technique and quasilinearization for some anti-periodic problems," Nonlinear World, vol. 3, no. 2, pp. 253-266, 1996.

[14] Y. Yin, "Remarks on first order differential equations with anti-periodic boundary conditions," Nonlinear Times and Digest, vol. 2, no. 1, pp. 83-94, 1995.

[15] A. R. Aftabizadeh, S. Aizicovici, and N. H. Pavel, "On a class of second-order anti-periodic boundary value problems," Journal of Mathematical Analysis and Applications, vol. 171, no. 2, pp. 301-320, 1992.

[16] S. Aizicovici, M. McKibben, and S. Reich, "Anti-periodic solutions to nonmonotone evolution equations with discontinuous nonlinearities," Nonlinear Analysis, vol. 43, pp. 233-251, 2001.

[17] Y. Chen, J. J. Nieto, and D. O’Regan, "Anti-periodic solutions for fully nonlinear first-order differential equations," Mathematical and Computer Modelling, vol. 46, no. 9-10, pp. 1183-1190, 2007.

[18] T. Y. Chen, W. B. Liu, J. J. Zhang, and M. Y. Zhang, "Existence of anti-periodic solutions for Liénard equations," Journal of Mathematical Study, vol. 40, no. 2, pp. 187-195, 2007 (Chinese).

[19] B. Liu, "Anti-periodic solutions for forced Rayleigh-type equations," Nonlinear Analysis, vol. 10, no. 5, pp. 2850-2856, 2009.

[20] W. Wang and J. Shen, "Existence of solutions for anti-periodic boundary value problems," Nonlinear Analysis, vol. 70, no. 2, pp. 598-605, 2009.

[21] Y. Li and L. Huang, "Anti-periodic solutions for a class of Liénard-type systems with continuously distributed delays," Nonlinear Analysis, vol. 10, no. 4, pp. 2127-2132, 2009.

[22] F.-J. Delvos and L. Knoche, "Lacunary interpolation by antiperiodic trigonometric polynomials," BIT, vol. 39, no. 3, pp. 439-450, 1999.

[23] J. Y. Du, H. L. Han, and G. X. Jin, "On trigonometric and paratrigonometric Hermite interpolation," Journal of Approximation Theory, vol. 131, no. 1, pp. 74-99, 2004.

[24] H. L. Chen, "Antiperiodic wavelets," Journal of Computational Mathematics, vol. 14, no. 1, pp. 32-39, 1996.

[25] G. Peng and L. Huang, "Anti-periodic solutions for shunting inhibitory cellular neural networks with continuously distributed delays," Nonlinear Analysis, vol. 10, no. 4, pp. 2434-2440, 2009.

[26] C. Ou, "Anti-periodic solutions for high-order Hopfield neural networks," Computers \& Mathematics with Applications, vol. 56, no. 7, pp. 1838-1844, 2008.

[27] S. Aizicovici, M. McKibben, and S. Reich, "Anti-periodic solutions to nonmonotone evolution equations with discontinuous nonlinearities," Nonlinear Analysis, vol. 43, pp. 233-251, 2001.

[28] J. Y. Shao, "Anti-periodic solutions for shunting inhibitory cellular neural networks with time-varying delays," Physics Letters, Section A, vol. 372, no. 30, pp. 5011-5016, 2008.

[29] Y. K. Li and L. Yang, "Anti-periodic solutions for Cohen-Grossberg neural networks with bounded and unbounded delays," Communications in Nonlinear Science and Numerical Simulation, vol. 14, no. 7, pp. 3134-3140, 2009.

[30] S. Gong, "Anti-periodic solutions for a class of Cohen-Grossberg neural networks," Computers $\mathcal{E}$ Mathematics with Applications, vol. 58, no. 2, pp. 341-347, 2009.

[31] M. Bohner and A. Peterson, Dynamic Equations on Time Scales, Birkhäuser, Boston, Mass, USA, 2001.

[32] M. Bohner and A. Peterson, Advances in Dynamic Equations on Time Scales, Birkhäuser, Boston, Mass, USA, 2003.

[33] V. Lakshmikantham and A. S. Vatsala, "Hybrid systems on time scales," Journal of Computational and Applied Mathematics, vol. 141, no. 1-2, pp. 227-235, 2002.

[34] E. R. Kaufmann and Y. N. Raffoul, "Periodic solutions for a neutral nonlinear dynamical equation on a time scale," Journal of Mathematical Analysis and Applications, vol. 319, no. 1, pp. 315-325, 2006.

[35] R. Agarwal, M. Bohner, and A. Peterson, "Inequalities on time scales: a survey," Mathematical Inequalities \& Applications, vol. 4, no. 4, pp. 535-557, 2001.

[36] M. Bohner, M. Fan, and J. Zhang, "Existence of periodic solutions in predator-prey and competition dynamic systems," Nonlinear Analysis, vol. 7, no. 5, pp. 1193-1204, 2006.

[37] D. Oregan, Y. J. Cho, and Y. Q. Chen, Topological Degree Theory and Application, Taylor \& Francis, London, UK, 2006.

[38] Y. K. Li, X. R. Chen, and L. Zhao, "Stability and existence of periodic solutions to delayed CohenGrossberg BAM neural networks with impulses on time scales," Neurocomputing, vol. 72, no. 7-9, pp. 1621-1630, 2009. 